\title{
Técnicas de preservación de la fertilidad femenina y experiencia en la Clínica de Fertilización Asistida en el Centro Médico ABC
}

\author{
Female fertility preservation techniques and our experience \\ in the Centro Medico ABC Fertility Center
}

\author{
Rolando Álvarez Valero, ,, Mónica Monzón Vargas, $\$$ Melissa Itzel Villagómez Alemán, \\ Julio González Cofrades, ${ }^{\ddagger}$ Eric Emilio Vázquez Camacho, ${ }^{\ddagger}$ Carlos Navarro Martínez ${ }^{\ddagger}$
}

Citar como: Álvarez VR, Monzón VM, Villagómez AMI, González CJ, Vázquez CEE, Navarro MC. Técnicas de preservación de la fertilidad femenina y experiencia en la Clínica de Fertilización Asistida en el Centro Médico ABC. An Med (Mex). $2020 ; 65$ (4): $280-287$. https://dx.doi.org/10.35366/97466

\section{RESUMEN}

La fertilidad femenina disminuye gradualmente desde la etapa embrionaria, se incrementa al inicio de la menarca y decrece nuevamente de manera significativa después de los 37 años. La preservación de fertilidad consiste en intervenciones médicas y/o quirúrgicas destinadas a proteger la fertilidad de pacientes cuya capacidad de reproducción se ve amenazada, ya sea por el envejecimiento ovárico natural o por diferentes condiciones médicas que disminuyen o comprometen la reserva ovárica. Gracias a los avances biotecnológicos en el área de reproducción asistida, actualmente es posible prevenir la pérdida de fertilidad con excelentes resultados (aumento en el número de $\mathrm{RN}$ ) obtenidos de la criopreservación de embriones, oocitos y/o tejido ovárico. Las pacientes candidatas para el uso de estas técnicas deben contar con una atención médica multidisciplinaria que permita individualizar su abordaje, por lo que es de vital importancia que el médico conozca las opciones de tratamiento existentes para brindar orientación oportuna. En este artículo se revisan las técnicas de preservación de fertilidad más

\section{ABSTRACT}

Female fertility decreases gradually from the embryonic stage, increasing at the onset of menarche and declining again significantly after 37 years. The preservation of fertility consists of medical and / or surgical interventions designed to protect the fertility of patients in which their reproductive capacity is threatened by the natural aging of the ovaries or by different medical conditions that diminish or compromise the ovarian reserve. Thanks to biotechnological advances in the area of assisted reproduction, it is now possible to prevent the loss of fertility with excellent results (increase in the number of newborns) obtained from the cryopreservation of embryos, oocytes and / or ovarian tissue. Patients who are candidates for this kind of techniques must have a multidisciplinary medical care that allows them to individualize their approach so it's of extreme importance that physicians are acquainted with the available treatment options to provide timely guidance. This article reviews the most important fertility preservation techniques, specific indications, effectiveness and safety, ending

\footnotetext{
* Human Care Medical Associates, S.A. de C.V. México.

‡ Clínica de Fertilización Asistida. Centro Médico ABC. México.

$\S$ Médico Residente Ginecología y Obstetricia. Centro Médico ABC. México.
}

Correspondencia: Dr. Rolando Álvarez Valero

Av. Carlos Graef Fernández Núm. 154, Col. Tlaxala, 05300,

Cuajimalpa de Morelos, CDMX, México.

Teléfonos: 55 1664-7305 / 55 1664-7306

E-mail: recepcion.mydoctor@gmail.com
Recibido para publicación: 23/03/2018. Aceptado: 19/11/2019.

\author{
Abreviaturas: \\ ASRM = Sociedad Americana de Medicina de la Reproducción. \\ HAM = Hormona antimülleriana \\ $\mathrm{HLA}=$ Antígeno leucocitario humano. \\ TRA = Técnicas de reproducción asistida . \\ $\mathrm{RN}=$ Recién nacido . \\ ICSI = Inyección intracitoplásmica de espermatozoides.
}


importantes, indicaciones específicas, efectividad y seguridad, finalizando con un análisis de la experiencia obtenida en un lapso de ocho años en la Clínica de Fertilización Asistida en el Centro Médico ABC.

Palabras clave: Fertilidad, preservación, criopreservación, reserva ovárica, gonadotóxicos.

Nivel de evidencia: III

\section{INTRODUCCIÓN}

Los ovarios cuentan con un número limitado de células germinales que tras su destrucción o atresia fisiológica no se regeneran. El número máximo de oocitos lo encontramos en el feto femenino a las 20 semanas de gestación con siete millones en promedio, al nacer sobreviven dos millones, en la menarca tan sólo restarán entre 300,000 y 500,000, de los cuales se pierden mes a mes 1,500 y para la menopausia hay sólo 100,000 . La mujer puede ovular en promedio 400 veces en su vida reproductiva, es decir, aprovecha un máximo de $0.08 \%$ de su reserva ovárica en la etapa reproductiva con estos fines. ${ }^{1,2}$

Contamos con diversas técnicas para la preservación de fertilidad que en conjunto con las TRA han sido un campo muy estudiado, y mantienen una constante evolución desde hace ya un par de décadas. Sin embargo, dichas técnicas siguen siendo poco conocidas y por ende subutilizadas, aun entre especialistas que ocupan de manera rutinaria fármacos gonadotóxicos en pacientes en edad fértil y con deseos genésicos no satisfechos. ${ }^{3-6}$

Por lo anterior, el objetivo principal de este artículo es resumir y exponer las diferentes opciones de tratamiento para prevenir la pérdida de fertilidad femenina haciendo énfasis en las opciones de criopreservación más utilizadas y que tenemos al alcance en nuestro medio. No se hablará de temas relacionados con cirugías conservadoras de fertilidad ni del uso de análogos de $\mathrm{GnRH}$ para prevenir gonadotoxicidad. 7,8

\section{Indicaciones para la prevención de la pérdida de fertilidad}

Existen condiciones médicas en las que deben considerarse técnicas de preservación de fertilidad, a continuación un listado de las más representativas:

- Cáncer (por efecto gonadotóxico de quimioterapia y radioterapia), se estima que hasta $25 \%$ de los casos de cáncer que afectan a la mujer se presen- with an analysis of the experience gained over a period of 8 years in the Fertility Center of the ABC Medical Center.

Keywords: Fertility, preservation, cryopreservation, ovarian reserve, gonadotoxic.

Level of evidence: III

tan en pacientes que no han iniciado su vida reproductiva. ${ }^{9,10}$

- Remoción quirúrgica o daño a órganos reproductores (resección de tumoraciones complejas o de gran tamaño como endometriomas o teratomas bilaterales). ${ }^{11}$

- Enfermedades autoinmunes (uso de agentes alquilantes inmunosupresores como ciclofosfamida) en lupus eritematoso sistémico, artritis reumatoide, dermatomiositis, entre otras. ${ }^{12,13}$

- Enfermedades hematopoyéticas, previo a trasplante de células madre se utilizan tanto quimioterapia como radioterapia para destruir células de la médula ósea preexistentes. ${ }^{7,14}$

- Alteraciones médicas que cursan con falla ovárica prematura: síndrome de Turner, síndrome de X frágil y galactosemia clásica.

- Social o «criopreservación electiva», mujeres que retrasan la maternidad por razones profesionales y/o personales a edades avanzadas, ${ }^{15}$ a este grupo también se le conoce como social freezing group. ${ }^{16,17}$

La última indicación continúa siendo un tema controversial, no existe información suficiente para recomendar la criopreservación con el único propósito de eludir el envejecimiento reproductivo en mujeres sanas; sin embargo, es evidente el beneficio que representa la criopreservación a edades tempranas evitando el riesgo de aneuploidía de oocitos envejecidos. ${ }^{18-21}$

En algunos países, hasta $30 \%$ de las mujeres podría considerar la criopreservación electiva como método para alargar su vida fértil. ${ }^{22,23}$

\section{Opciones para la preservación de la fertilidad femenina}

Los procedimientos para la preservación de la fertilidad femenina más estudiados y con mayor relevancia a nivel mundial son:

a) Criopreservación de embriones: técnica que cuenta con mayor experiencia, desventaja en cuanto a 
problemáticas legales y éticas, se requiere consentimiento de la pareja y apegarse a las regulaciones de cada país.

b) Criopreservación de oocitos maduros: la ventaja que representa es la autonomía reproductiva que le ofrece a la paciente. ${ }^{24}$

c) Criopreservación de tejido ovárico: considerada aún como técnica experimental; sin embargo, representa la mejor opción para niñas prepúberes. ${ }^{1,25}$

\section{Diferencias entre procedimientos de criopreservación: ventajas y desventajas \\ Criopreservación de embriones}

En 1983 Trounson \& Mohr ${ }^{26}$ describen con éxito la transferencia de embriones descongelados. La criopreservación de embriones es la técnica que cuenta con mayor experiencia y tasas de efectividad demostradas desde hace más de 30 años. ${ }^{27,28}$ Una de sus principales desventajas es la problemática legal, ya que requiere del consentimiento de ambos padres biológicos para su uso, sin dejar de lado la cuestión ética que supone el futuro incierto de embriones abandonados. ${ }^{29}$

\section{Criopreservación de oocitos}

El primer nacimiento producto de un oocito congelado fue en 1986; sin embargo, es en esta última década que esta técnica ha ganado popularidad.

La desventaja es que para la obtención de oocitos se requieren de por lo menos dos semanas en promedio para la realización de la estimulación ovárica controlada y la recolección, lo que puede retrasar el inicio de tratamiento oncológico o inmunosupresor. Un ejemplo claro es el cáncer de mama en el que se ha calculado una disminución en la sobrevida de $15 \%$ por cada cuatro semanas de retraso en el inicio de quimioterapia. ${ }^{30,31}$

Existen dos tipos de criopreservación de oocitos:

- Técnica de enfriamiento lento o también conocida como tasa controlada de criopreservación (técnica convencional), en la cual se va disminuyendo la temperatura de manera progresiva hasta llegar por debajo del punto de congelación en presencia de bajas concentraciones de agentes citoprotectores (azúcares, propanediol). Desventajas: daño estructural, formación de cristales de hielo durante el enfriamiento. ${ }^{32}$
- Técnica de enfriamiento ultra-rápido conocida como vitrificación. Es el proceso mediante el cual existe transformación de líquido a sólido en ausencia de cristalización. De esta forma se disminuye el riesgo de daño criogénico, a la vez que se evitan lesiones osmóticas y tóxicas sobre la estructura e integridad genética. Se requieren altas concentraciones de agentes crioprotectores (etilenglicol, dimetilsulfóxido, sucrosa) y posterior enfriamiento 1,000 veces más rápido.

Desde 2012 la Sociedad Americana de Medicina de la Reproducción (ASRM) eliminó la designación de experimental a esta técnica de criopreservación de oocitos, pero en 2013 se publicó como parte de la guía de práctica clínica de la ASMR, en Fertility and Sterility. ${ }^{33}$

Existen dos sistemas de vitrificación: sistema abierto que utiliza nitrógeno líquido y un sistema cerrado que se enfría más lento y requiere de mayor concentración de crioprotectores, pero que a diferencia del abierto protege de una posible contaminación. ${ }^{34}$

Desventajas: se han documentado alteraciones en el huso mitótico secundario al uso de altas concentraciones de crioprotectores y también se ha visto que el nitrógeno líquido puede alterar la arquitectura sensible de oocitos maduros sin que esto represente una significancia en los resultados clínicos como explicaremos a continuación.

Cuando se comparó la utilización de oocitos frescos autólogos vs. vitrificados se obtuvieron tasas similares de embarazos para ambos grupos. Cobo $^{35}$ y colaboradores compararon 996 embarazos producto de oocitos frescos vs. 804 embarazos producto de oocitos vitrificados. No existió diferencia en las bases demográficas, factores de riesgo o condiciones maternas (excepto que se encontró una edad más avanzada en el segundo grupo). Los resultados fueron similares en cuanto a complicaciones relacionadas al embarazo así como desenlaces obstétricos y neonatales.

Las tasas similares de fertilización, implantación, embarazo, aborto y número de recién nacidos vivos producto de oocitos frescos vs. vitrificados sugiere no sólo la integridad morfológica, sino también la funcional. ${ }^{36,37}$

La vitrificación ha mostrado tasas de embarazos superiores a la técnica de enfriamiento lento así como mayor supervivencia de oocitos congelados, por lo que actualmente se considera la técnica de elección. 18,38

Un metaanálisis que incluyó 14 estudios controlados, aleatorizados que compararon ambas técnicas sugiere que la vitrificación puede ser más efectiva causando menos daño a folículos primordiales así 
como menor tasa de rupturas en el ADN y mejor preservación de células estromales. ${ }^{32}$

En pacientes menores de 38 años se calcula una tasa de éxito (logrando un RN con técnica ICSI) de alrededor de $46 \%$ con la vitrificación de ocho ovocitos y de $70-80 \%$ cuando se vitrifican $15-20$ ovocitos. ${ }^{39}$

Vale la pena mencionar que existen diferentes resultados en la literatura al utilizar oocitos autólogos vs. heterólogos. Esto es de esperarse, ya que en cada caso influye tanto la edad materna como el motivo de la criopreservación (enfermedades de base).

Los principales factores para estimar la probabilidad de lograr un nacimiento producto de oocitos criopreservados son:

- Número total de oocitos maduros criopreservados.

- Edad materna en el momento de la recolección (ya que la tasa de eficiencia disminuye con la edad de forma sustancial en pacientes mayores de 37 años). ${ }^{40}$

\section{Criopreservación de tejido ovárico}

En niñas prepúberes que no han ovulado todavía y que no son candidatas a criopreservación de oocitos maduros, una alternativa es la criopreservación de tejido ovárico. No se requiere de la utilización de ultrasonido endovaginal ni de la estimulación ovárica controlada, tan solo se necesita la extracción de suficiente corteza ovárica para su criopreservación, lo que se puede realizar con técnicas quirúrgicas de mínima invasión. ${ }^{41,42}$

Por otro lado, en mujeres con procesos oncológicos agresivos de tipo hormono-dependiente y en aquellos casos en los que no es urgente iniciar el tratamiento médico con potencial gonadotoxicidad, se puede utilizar esta técnica de preservación de fertilidad de forma rápida y eficaz. ${ }^{5,28,43}$

Cada vez contamos con mayor evidencia de la ventaja que representa la criopreservación de tejido ovárico para la restauración de la función endócrina y para lograr embarazos ya sean espontáneos o bien producto de TRA, lo que apoya su futura consideración como aplicación clínica y que al igual que la vitrificación en su momento dejará de ser considerada un procedimiento experimental. ${ }^{44}$

Hasta la fecha se han reportado alrededor de 100 nacimientos vivos alrededor del mundo producto de esta técnica, teniendo una tasa de éxito reportada de $30 \%$ posterior al autotrasplante de corteza ovárica. ${ }^{43,45}$ El primer caso de un nacimiento vivo tras un autotransplante fue en 2004, publicado por Donnez y colaboradores; sin embargo, aún son pocas las publicaciones que reportan su efectividad. ${ }^{46}$

Existen dos tipos de trasplante de tejido ovárico: el ortotópico que es la recolocación en cavidad pélvica, específicamente sobre médula de ovario restante, y el heterotópico (fuera de la cavidad), ${ }^{47}$ en el que se coloca en tejido celular subcutáneo de abdomen y antebrazo. El primero es compatible con reproducción natural, mientras que el segundo requiere de fecundación in vitro. ${ }^{43,48}$

La principal desventaja se presenta en pacientes con procesos oncológicos, ya que en el caso de trasplante de tejido ovárico autólogo existe la posibilidad de reimplantación de células cancerígenas originales. ${ }^{9}$

La posibilidad de maduración in vitro de oocitos del tejido ovárico criopreservado acompañado de inseminación in vitro o ICSI permite a este grupo particular de pacientes embarazarse sin tener que efectuar el trasplante de tejido ovárico. ${ }^{49-51}$ Se han documentado 5,000 nacimientos con el uso de esta técnica. ${ }^{39}$

Tabla 1: Clasificación de gonadotóxicos. 3,22,23,28,33,52

\begin{tabular}{|c|c|}
\hline Clasificación de agentes gonadotóxicos & Ejemplos \\
\hline Alto riesgo & Ciclofosfamida, clorambucilo, melfalán, busulfán, mostaza nitrogenada, procarbazina \\
\hline Riesgo intermedio & Cisplatino, carboplatino y adriamicina \\
\hline Bajo riesgo & Metotrexato, 5-fluorouracilo, vincristina, bleomicina, actinomicina D \\
\hline \multicolumn{2}{|c|}{$\begin{array}{l}\text { Radioterapia: dosis dependiente, }<4 \text { Gy destruye } 50 \% \text { de la población oocitaria } \\
\text { Dosis letal: } 50 \mathrm{~Gy}\end{array}$} \\
\hline \multirow{4}{*}{ Riesgo desconocido } & Trastuzumab, cetuximab (anticuerpos monoclonales) \\
\hline & Paclitaxel, docetaxel (familia de taxanos) \\
\hline & Irinotecán \\
\hline & Rapamicina \\
\hline
\end{tabular}




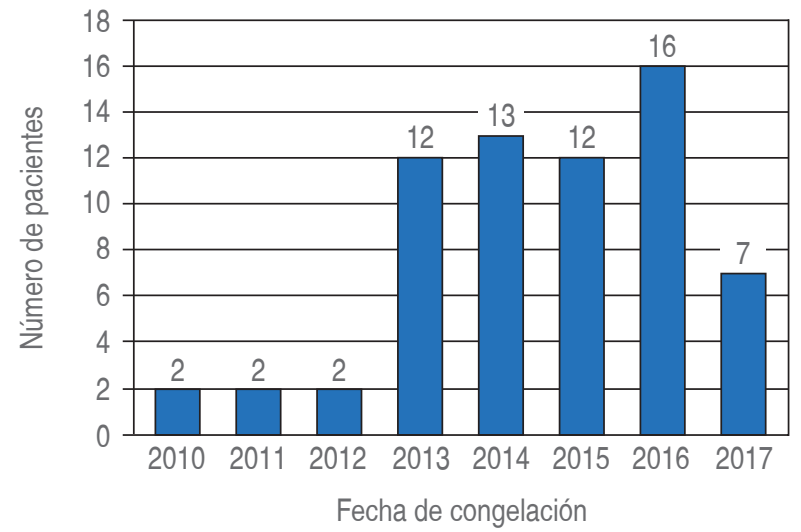

Figura 1: Pacientes con criopreservación de oocitos en la Clínica de Fertilidad del Centro Médico ABC.

\section{Predictores del resultado}

Es esencial estimar el éxito individualizado de las técnicas propuestas. Los marcadores de reserva ovárica que más se utilizan son la HAM (hormona antimülleriana) y conteo de folículos antrales, una HAM $<1.2 \mathrm{ng} / \mathrm{mL}$ y $<4$ folículos antrales acompañados de un volumen ovárico $<3 \mathrm{~cm}^{3}$, nos habla de una respuesta deficiente ante cualquier método preservador de la fertilidad; sin embargo, la edad materna sigue siendo el factor limitante más importante (independiente de los niveles de FSH). ${ }^{2}$

El predictor más confiable para calcular la eficacia en la obtención de un nacimiento vivo por oocito congelado es la edad, siendo de: $8.7 \%$ para mujeres menores de 30 años y de $1.1 \%$ en mujeres entre 43 y 44 años. ${ }^{1,30}$

Respecto al número de ovocitos que se deben obtener para lograr mejores resultados, en un estudio realizado en el INPER (Instituto Nacional de Perinatología) mediante el análisis de 673 ciclos se concluyó que un número mayor o igual a 12 ovocitos recolectados representa mayores tasas de lograr un RN. ${ }^{53}$

\section{Oncofertilidad}

Hoy en día la preservación de la fertilidad no es un hábito que se practique con frecuencia, aun en países desarrollados como Estados Unidos y el Reino Unido se estima que entre $30-60 \%$ de supervivientes de cáncer no recibieron información sobre el tema, mismas cifras que se incrementan en países en vías de desarrollo.

En una encuesta realizada en Suiza, más de 50\% de pacientes supervivientes de alguna enfermedad oncológica refirieron no haber recibido asesoría previa al inicio de su tratamiento sobre técnicas para la preservación de la fertilidad. ${ }^{28,54}$

Ante estas circunstancias ha surgido una nueva rama de estudio: «la oncofertilidad», la cual busca abordar este tipo de problemas. ${ }^{9}$

Se menciona la importancia de proveer información a la paciente con respecto al envejecimiento ovárico fisiológico, el efecto que suponen tratamientos oncológicos y/o inmunosupresores sobre su fertilidad así como los diferentes métodos de preservación de fertilidad actuales, su eficacia, límites y los desafíos relativos a su aplicación. ${ }^{55,56}$

Igualmente el médico debe tener una noción general de los fármacos capaces de alterar la fertilidad y conocer su clasificación dependiendo de la toxicidad que ejercen sobre los gametos para poder aconsejar de manera correcta y oportuna a sus pacientes. ${ }^{57}$ En la Tabla 1 enumeramos los principales medicamentos gonadotóxicos.

Asimismo, es importante el aspecto económico, ya que no existen estrategias de salud pública orientadas a la preservación de la fertilidad, y en países como México y Chile la mayoría de los ciclos con TRA realizados son financiados por las propias parejas. ${ }^{58}$

\section{EXPERIENCIA EN LA CLÍNICA DE FERTILIDAD DEL CENTRO MÉDICO ABC}

Desde el año 2010 se ha llevado a cabo la criopreservación de oocitos maduros en nuestra clínica de 66 pacientes mediante técnica de vitrificación abierta. Se observa un aumento importante en el número de pacientes que se sometieron a dicha técnica a partir de 2013, año en el que deja de ser un procedimiento experimental y es aprobado como método clínico eficaz para la criopreservación de la fertilidad.

Los datos presentados abarcan desde 2010 hasta principios del mes de septiembre de 2017, con una recolección total de 551 oocitos (Figura 1).

Tabla 2: Indicación de criopreservación en la Clínica de Fertilidad del Centro Médico ABC.

\begin{tabular}{lcccc}
\hline \multicolumn{4}{c}{ Indicación de criopreservación } \\
\hline & $\begin{array}{c}\text { Número de } \\
\text { pacientes }\end{array}$ & $\begin{array}{c}\text { Porcentaje de } \\
\text { pacientes }\end{array}$ & $\begin{array}{c}\text { Número } \\
\text { de óvulos }\end{array}$ & $\begin{array}{c}\text { Porcentaje } \\
\text { de óvulos }\end{array}$ \\
\hline Oncofertilidad & 9 & 25 & 19 & 4 \\
Otros & 50 & 85 & 465 & 96 \\
Total & 59 & 100 & 484 & 100 \\
\hline
\end{tabular}




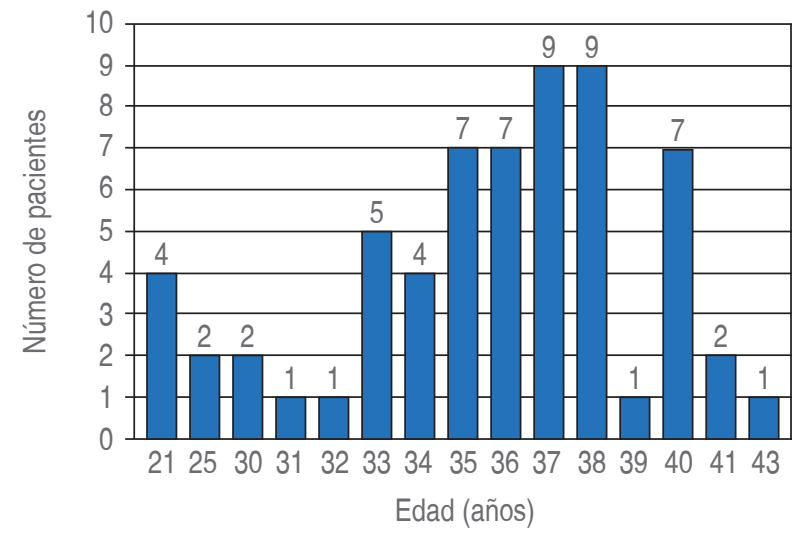

Figura 2: Distribución de pacientes por edad.

Se excluyeron cuatro pacientes, en las que no se obtuvo la información completa como la edad y/o indicación médica para la criopreservación de óvulos.

En nuestros resultados encontramos que las indicaciones principales para criopreservación en nuestro medio fueron: edad, factores como baja reserva ovárica y resección de tumores ováricos complejos, los cuales se incluyeron en la categoría «Otros» y corresponden a $85 \%$ del total. El restante $25 \%$ corresponde a pacientes que estaban por iniciar tratamiento oncológico con fármacos potencialmente gonadotóxicos (Tabla 2 y Figura 2).

Por último, se muestra un histograma con la distribución de pacientes por grupo de edades, encontrando que los grupos de edades con mayor número de pacientes que se sometieron a criopreservación de oocitos maduros fueron los de 37 y 38 años con nueve pacientes cada uno, el segundo lugar lo ocupan los grupos de 35, 36 y 40 años, con siete pacientes cada uno. En el último lugar se encuentran las pacientes de $31,32,39$ y 41 años, con una paciente en cada grupo.

\section{DISCUSIÓN}

Existen diversos estudios que garantizan como opción viable y segura la criopreservación por vitrificación como método de preservación de la fertilidad; sin embargo, continúan siendo muy pocas las pacientes en nuestro país que aun teniendo una indicación médica específica, son derivadas a especialistas en medicina de la reproducción y fertilidad.

El número de pacientes que se someten a criopreservación de oocitos maduros en la Clínica de Fertilidad del Centro Médico ABC va en ascenso, el análisis de las tasas de éxito de embarazos y nacimientos será motivo de un segundo análisis.

\section{CONCLUSIÓN}

Es importante promover una cultura de preservación de la fertilidad teniendo en cuenta que la edad óptima para criopreservar en aquellas mujeres que desean posponer la maternidad es antes de los 37 años, sin olvidar que los riesgos maternos se incrementan conforme avanza la edad materna.

No debemos perder de vista que si bien los tratamientos oncológicos e inmunosupresores son la prioridad en el manejo médico, el deseo reproductivo individualizado no debe dejarse de lado. Tanto los médicos como la población general deben tener conocimiento sobre la importancia y las posibilidades existentes en nuestro medio para preservar la fertilidad femenina a futuro.

Deberán gestionarse nuevas estrategias de salud pública en nuestro país que incluyan la preservación de la fertilidad de sus derechohabientes y que se puedan ofrecer a toda la población.

\section{BIBLIOGRAFÍA}

1. Gris Martínez JM, Callejo Olmos J, Pérez Milán F. Preservation of fertility in women undergoing cytotoxic therapies. Med Clin (Barc). 2011; 137 (15): 702-707.

2. Cabry R, Merviel P, Hazout A, Belloc S, Dalleac A, Copin H et al. Management of infertility in women over 40 . Maturitas. 2014; 78 (1): 17-21. doi: 10.1016/j.maturitas.2014.02.014.

3. Garrido-Colino C, Lassaletta A, Vazquez MA, Echevarria A, Gutierrez I, Andión M et al. Current situation on fertility preservation in cancer patients in Spain: Level of knowledge, information, and professional involvement. An Pediatr (Barc). 2017; 87 (1): 3-8.

4. Woodard TL, Hoffman AS, Covarrubias LA, Holman D, Schover L, Bradford A et al. The Pathways fertility preservation decision aid website for women with cancer: development and field testing. J Cancer Surviv. 2018; 12 (1): 101-114.

5. Jones G, Hughes J, Mahmoodi N, Smith E, Skull J, Ledger W. What factors hinder the decision- making process for women with cancer and contemplating fertility preservation treatment? Hum Reprod Update. 2017; 23 (4): 433-457.

6. Algarroba GN, Sanfilippo JS, Valli-Pulaski H. Female fertility preservation in the pediatric and adolescent cancer patient population. Best Pract Res Clin Obstet Gynaecol. 2018; 48: 147-157.

7. Anderson RA, Wallace WHB, Telfer EE. Ovarian tissue cryopreservation for fertility preservation: clinical and research perspectives. Hum Reprod Open. 2017; 2017 (1): hox001. doi: 10.1093/hropen/hox001.

8. Lambertini M, Ceppi M, Poggio F, Peccatori FA, Azim HA Jr, Ugolini D et al. Ovarian suppression using luteinizing hormone-releasing hormone agonists during chemotherapy to preserve ovarian function and fertility of breast cancer patients: a meta-analysis of randomized studies. Ann Oncol. 2015; 26 (12): 2408-2419. doi: 10.1093/annonc/mdv374.

9. Zapardiel I, Diestro MD, Aletti G. Conservative treatment of early stage ovarian cancer: oncological and fertility outcomes. Eur J Surg Oncol. 2014; 40 (4): 387-393. doi: 10.1016/j. ejso.2013.11.028. 
10. Moravek MB, Confino R, Smith KN, Kazer RR, Klock SC, Lawson AK et al. Long-term outcomes in cancer patients who did or did not pursue fertility preservation. Fertil Steril. 2018; 109 (2): 349-355.

11. De la Fuente L, Ortega S, Monzó A, Martín B, Iñarra MJ, Hernández $\mathrm{C}$ et al. Preservación de la fertilidad en endometriosis: estado actual de conocimiento y papel del sistema público de salud. Medicina Reproductiva y Embriología Clínica. 2016; 3 (3): 119-127.

12. Martinez F; International Society for Fertility PreservationESHRE-ASRM Expert Working Group. Update on fertility preservation from the Barcelona International Society for Fertility Preservation-ESHRE-ASRM 2015 expert meeting: indications, results and future perspectives. Fertil Steril. 2017; 108 (3): 407-415.e11.

13. Steta-Mondragón JR, Valero OPA, Deschamps-Díaz H, Álvarez-Valero R. Teratoma bilateral y preservación de la fertilidad. An Med Asoc Med Hosp ABC. 2012; 57 (4): 325-331.

14. Rodríguez GAN, Osorio SSB, Maldonado VMR, Faugier FE. Lupus eritematoso generalizado juvenil: patrones clínicos e inmunológicos en una cohorte de 150 pacientes del Hospital Infantil de México «Federico Gómez». An Med Asoc Med Hosp ABC. 2016; 61 (3): 182-187.

15. Lugones Botell M. Preserving fertility from primary health care. Rev Cuba Obstet Ginecol. 2014; 40 (3): 301-302.

16. Qiao J, Li R. Fertility preservation: challenges and opportunities. Lancet. 2014; 384 (9950): 1246-1247. doi: 10.1016/S0140-6736(14)61749-9.

17. Menjón BS. ¿Debemos incorporar la preservación de la fertilidad en el protocolo diagnóstico-terapéutico del cáncer? Rev Senol Patol Mamar. 2017; 30 (1): 1-2.

18. Garcia-Velasco JA, Domingo J, Cobo A, Martínez M, Carmona L, Pellicer A. Five years' experience using oocyte vitrification to preserve fertility for medical and nonmedical indications. Fertil Steril. 2013; 99 (7): 1994-1999.

19. Soriano-Ortega KP, Carballo-Mondragón E, Roque-Sánchez AM, Durán-Monterrosas L, Kably-Ambe A. Percepción de la fertilidad en mujeres en edad reproductiva, según su edad. Ginecol Obstet Mex. 2017; 85 (6): 364-373.

20. Ramos RR, Gutiérrez GR, Monroy IA, Sánchez HG. Factores de riesgo asociados con infertilidad femenina. Ginecol Obstet Mex. 2008; 76 (12): 717-721.

21. Brugo-Olmedo S, Chillik C, Kopelman S. Definición y causas de la infertilidad. Rev Colomb Obstet Ginecol. 2003; 54 (4): 227-248.

22. De Munck N, Vajta G. Safety and efficiency of oocyte vitrification. Cryobiology. 2017; 78: 119-127. doi: 10.1016/j. cryobiol.2017.07.009.

23. Santo EVE, Dieamant F, Petersen CG, Mauri AL, Vagnini LD, Renzi A et al. Social oocyte cryopreservation: a portrayal of Brazilian women. JBRA Assist Reprod. 2017; 21 (2): 101-104.

24. Kutluk Oktay, MD, FACOG. Fertility preservation: we are in this a long haul. Editotial. Am J Obstet Gynecol. 2013: 77-79.

25. Pouly JL, Brugnon F, Pons H, Dejardin M, Tartiere M, Gremeau AS et al. Donación de gametos y de embriones. EMC -Ginecología - Obstetricia. 2014; 50 (1): 1-14.

26. Trounson A, Mohr L. Human pregnancy following cryopreservation, thawing and transfer of an eight-cell embryo. Nature. 1983; 305 (5936): 707-709.

27. Camus A. Preservación de la fertilidad en la mujer. Rev Med Clin Condes. 2010; 21 (3): 440-449.

28. Rodriguez-Wallberg KA, Oktay K. Options on fertility preservation in female cancer patients. Cancer Treat Rev. 2012; 38 (5): 354-361.
29. Almodin CG, Ceschin A, Nakano RE, Radaelli MR, Almodin PM, Silva CG et al. Vitrification of human oocytes and its contribution to in vitro fertilization programs. JBRA Assist Reprod. 2015; 19 (3): 135-140.

30. Massarotti C, Scaruffi P, Lambertini M, Remorgida V, Del Mastro L, Anserini P. State of the art on oocyte cryopreservation in female cancer patients: A critical review of the literature. Cancer Treat Rev. 2017; 57: 50-57.

31. De Vos M, Smitz J, Woodruff TK. Fertility preservation in women with cancer. Lancet. 2014; 384 (9950): 1302-1310. doi: 10.1016/S0140-6736(14)60834-5. Erratum in: Lancet. 2015; 385 (9971): 856.

32. Shi Q, Xie Y, Wang Y, Li S. Vitrification versus slow freezing for human ovarian tissue cryopreservation: a systematic review and meta-anlaysis. Sci Rep. 2017; 7 (1): 8538.

33. Practice Committees of the American Society for Reproductive Medicine and the Society for Assisted Reproductive Technology. Mature oocyte cryopreservation: a guideline. Fertil Steril. 2013; 99 (1): 37-43.

34. Gook DA, Choo B, Bourne H, Lewis K, Edgar DH. Closed vitrification of human oocytes and blastocysts: outcomes from a series of clinical cases. J Assist Reprod Genet. 2016; 33 (9): $1247-1252$.

35. Cobo A, Serra V, Garrido N, Olmo I, Pellicer A, Remohí J. Obstetric and perinatal outcome of babies born from vitrified oocytes. Fertil Steril. 2014; 102 (4): 1006-1015.e4.

36. Crawford S, Boulet SL, Kawwass JF, Jamieson DJ, Kissin DM. Cryopreserved oocyte versus fresh oocyte assisted reproductive technology cycles, United States, 2013. Fertil Steril. 2017; 107 (1): 110-118.

37. Ho JR, Woo I, Louie K, Salem W, Jabara SI, Bendikson KA et al. A comparison of live birth rates and perinatal outcomes between cryopreserved oocytes and cryopreserved embryos. J Assist Reprod Genet. 2017; 34 (10): 1359-1366.

38. Edgar DH, Gook DA. A critical appraisal of cryopreservation (slow cooling versus vitrification) of human oocytes and embryos. Hum Reprod Update. 2012; 18 (5): 536-554.

39. Comtet M, Benard J, Grynberg M. Preservación de la fertilidad femenina. EMC Ginecol-Obst. 2017; 53 (1): 1-15.

40. Álvarez A. ¿Cuál sería la edad máxima para recomendar la preservación de la fertilidad por indicación no médica (indicación social)? En: Bruna I, Coroleu B. Lo esencial en Medicina Reproductiva. 2a edición. Barcelona: Elsevier; 2017. pp. 238-240.

41. Haino T, Tarumi W, Kawamura K, Harada T, Sugimoto K, Okamoto A et al. Determination of follicular localization in human ovarian cortex for vitrification. J Adolesc Young Adult Oncol. 2018; 7 (1): 46-53.

42. Bastings L, Beerendonk CC, Westphal JR, Massuger LF, Kaal SE, van Leeuwen FE et al. Autotransplantation of cryopreserved ovarian tissue in cancer survivors and the risk of reintroducing malignancy: a systematic review. Hum Reprod Update. 2013; 19 (5): 483-506.

43. Kristensen SG, Giorgione V, Humaidan P, Alsbjerg B, Bjørn AB, Ernst E et al. Fertility preservation and refreezing of transplanted ovarian tissue-a potential new way of managing patients with low risk of malignant cell recurrence. Fertil Steril. 2017; 107 (5): 1206-1213.

44. Jadoul P, Guilmain A, Squifflet J, Luyckx M, Votino R, Wyns $\mathrm{C}$ et al. Efficacy of ovarian tissue cryopreservation for fertility preservation: lessons learned from 545 cases. Hum Reprod. 2017; 32 (5): 1046-1054.

45. Andersen CY. Success and challenges in fertility preservation after ovarian tissue grafting. Lancet. 2015; 385 (9981): 19471948. doi: 10.1016/S0140-6736(15)60960-6. 
46. Silber S. Ovarian tissue cryopreservation and transplantation: scientific implications. J Assist Reprod Genet. 2016; 33 (12): 1595-1603.

47. Di Pietro M, Virdis A, González FJ. Preservar la fertilidad en Pediatría a partir de la congelación de tejido ovárico: ¿Cuál es el mejor interés de la niña? Revista Pax et Emerita.2015; 11: 87-100.

48. Sánchez M, Edurne RE, Busso C, Pellice A. Inducción de la ovulación. Capítulo: Criopreservación y autotrasplante de tejido ovárico. España, S.L.: Elsevier; 2014.

49. Stachs A, Hartmann S, Gerber B. Preservation of fertility or ovarian function in patients with breast cancer or gynecologic and internal malignancies. Geburtshilfe Frauenheilkd. 2017; 77 (8): 861-869.

50. Álvarez A. ¿En qué casos está indicada la congelación de tejido ovárico en una preservación de la fertilidad?. En: Bruna I, Coroleu B. Lo esencial en medicina reproductiva. 2a edición. Barcelona: Elsevier; 2017. pp. 247-249.

51. Aguilar Melgar AE. Enfoque de la preservación de la fertilidad femenina. Rev Mex Reprod. 2013; 5: 157-158.

52. Lombardi EP, Sícaro LV. Preservación de la fertilidad. Rev Endocrinol Ginecol Reprod. 2003; 44-55.
53. López-Rioja MJ, Campos-Cañas JA, Recio-López Y, QuirozGarza G, Sánchez-González CM, Hinojosa-Rodríguez K et al. Optimal number of oocytes: in vitro fertilization predictive model. Ginecol Obstet Mex. 2017; 85 (11): 735747.

54. Medrano JV, Andrés MDM, García S, Herraiz S, VilanovaPérez T, Goossens E et al. Basic and clinical approaches for fertility preservation and restoration in cancer patients. Trends Biotechnol. 2018; 36 (2): 199-215. doi: 10.1016/j. tibtech.2017.10.010.

55. Urech C, Ehrbar V, Boivin J, Müller M, Alder J, Zanetti Dällenbach $R$ et al. Knowledge about and attitude towards fertility preservation in young female cancer patients: a crosssectional online survey. Hum Fertil (Camb). 2018; 21 (1): 45-51.

56. Gunnala V, Schattman G. Oocyte vitrification for elective fertility preservation: the past, present, and future. Curr Opin Obstet Gynecol. 2017; 29 (1): 59-63.

57. Lineamientos de infertilidad. Ginecol Obstet Mex. 2011; 79 (11): 659-673.

58. Schwarze JE, Zegers F, Galdames V. Reproducción asistida en Latinoamérica y Chile Rev Med Clin Condes. 2010; 21 (3): 451-456. 